\title{
Time-dependent release of extracellular vesicle subpopulations in tumor CABA I cells
}

\author{
ILARIA GIUSTI $^{1 *}$, MARIANNA DI FRANCESCO $^{1 *}$, LAURA CANTONE $^{2}$, SANDRA D'ASCENZO $^{1}$, \\ VALENTINA BOLLATI $^{2}$, GASPARE CARTA ${ }^{1}$ and VINCENZA DOLO ${ }^{1}$ \\ ${ }^{1}$ Department of Life, Health and Environmental Sciences, University of L'Aquila, L'Aquila; \\ ${ }^{2}$ Department of Clinical Sciences and Community Health, Molecular Epidemiology and \\ Environmental Epigenetics Laboratory, Università degli Studi di Milano, Milan, Italy
}

Received April 16, 2015; Accepted July 6, 2015

DOI: $10.3892 /$ or.2015.4199

\begin{abstract}
Investigations into extracellular vesicles (EVs) have significantly increased since their role in physiological and pathological processes has become more clearly understood. Furthermore, it has become increasingly clear that several subpopulations of EVs exist, such as exosomes (EXOs) and microvesicles (MVs). Various methods and techniques used to identify and isolate the specific EVs subpopulations exist. However, these methods should be further elucidated. A deep understanding of the different factors that affect the EVs release may therefore be useful for the standardization of protocols and to establish guidelines for a more adequate analysis and correct inter-laboratory comparison. In the present study, we investigated whether composition and molecular features of EVs altered over time following a trigger stimulus. Starved CABA I cells were stimulated with FBS and conditioned medium was collected after different time intervals (30 min and 4, 8 and $18 \mathrm{~h}$ ). The dynamic of EVs release was time-dependent, as shown by the results of scanning electron microscopy. Additionally, the time elapsed from the stimulus affected the size distribution (as highlighted by transmission electron microscopy and NanoSight assay), amount (in terms of the number of particles and protein amount) and molecular composition (CD63, HLA, Ago-2, gelatinases, and plasminogen activators) suggesting that, different EVs subpopulations were released at different time intervals following cell stimulation. Collectively, the results suggested that, parameters useful to standardize procedures for EVs isolation, including stimulation time should be considered.
\end{abstract}

Correspondence to: Professor Vincenza Dolo, Department of Life, Health and Environmental Sciences, University of L'Aquila, Via Vetoio-Coppito 2, I-67100, L'Aquila, Italy

E-mail: vincenza.dolo@univaq.it

*Contributed equally

Key words: extracellular vesicles, exosomes, microvesicles, ovarian cancer

\section{Introduction}

In the last two decades it has become clear that, besides the direct cell-to-cell contact and transfer of secreted molecules, there is an additional mechanism in intercellular communication involving extracellular vesicles (EVs) (1). It is well known that a variety of cells is able to secrete several types of EVs. When initially identified EVs were considered a form of waste elimination, whereas it has become clear that these membrane-enclosed structures are signaling packages able to interact with the extracellular matrix, by modifying it, and the surrounding cells, by stimulating or inhibiting these structures (2,3). EVs can transport several bioactive molecules, such as proteins, lipids and RNAs, and can propagate their content in recipient cells through horizontal transfer, thus having a significant impact on the surrounding extracellular matrix and cells (4).

Previous studies defined the importance of this novel mechanism of intercellular communication in various physiological and pathological events, such as the immune response, cell differentiation and vascular, neurological and cancer diseases $(2,5)$.

EVs are divided into three major groups represented by apoptotic bodies, exosomes (EXOs) and shed microvesicles (MVs). EXOs and MVs, which are released from viable cells, are considered to be primarily involved in the exchange of messages between cells (6). Additionally, they have a rounded shape and differ mainly in cell origin and size range. EXOs size ranges between 30 and $100 \mathrm{~nm}$ whereas that of MVs ranges between 100 and 1,000 nm (10). EXOs are released following fusion with the plasma membrane of multivesicular bodies (MVB), which belong to the endosomal compartment and form through a process of inward budding that gives origin to intraluminal vesicles (ILVs); once the ILVs are released from MVB into extracellular milieu they are called EXOs $(7,8)$. The origin of MVs is completely different since they are formed through the outward budding of plasma membrane $(2,7,8)$.

Exosomes have been mainly studied for their involvement in immune system functions but also for their role in cancer progression $(4,7,9)$. In the same manner, MVs have been widely studied in several normal cell types, including platelets, fibroblasts, neuronal, epithelial, endothelial and red 
blood cells (10-13), and even more widely analyzed in cancer cells $(6,14-18)$ for their role in tumor progression, evasion from apoptosis, drug resistance, immune-escape, and angiogenesis $(2,3,19)$.

The non-accidental production of EVs, in pathological and physiological events, is emphasized by the fact that they are not merely miniature parental cells, showing both similarities and differences compared to the molecular composition of the cells of origin $(6,20)$.

Due to their role in pathological processes, EVs have been widely studied in the past few years (21-24). Moreover, there is an increasing expectation in their potential use as clinical targets and as diagnostic and prognostic biomarkers since vesicles occur in bloodstream and other biological fluids (e.g., urine, semen, amniotic fluid, saliva, synovial and bronchoalveolar fluid, breast milk, spinal fluid, ascites, malignant and pleural effusion), particularly the ones that are exposed to primary tumors $(2,19,25-28)$.

Since in EXOs the upper limit of size overlaps with the MVs lower limit (being $100 \mathrm{~nm}$ for both) and molecular features are often shared, it is extremely difficult to strictly separate the two subpopulations with the currently available techniques. This explains the reason for most studies analyzing mixed populations or at best, populations specifically enriched with one component, but contaminated with another one (2).

Even if methods and techniques to identify and isolate the specific EVs subpopulations evolve continuously, this goal has not yet been fully achieved.

Information pertaining to the biology of EVs, including mechanisms involved in their release, may be useful to gain a better understanding of the phenomenon and pave the way for the identification of isolation strategies and standardization of protocols, allowing a more adequate analysis and correct inter-laboratory comparison.

\section{Materials and methods}

Cell culture. CABA I cells were used in all the experiments. The CABA I cell line was established from the ascitic fluid of an ovarian carcinoma patient not undergoing drug treatment (29).

The cells were grown as monolayers in RPMI-1640 containing $5 \%(\mathrm{v} / \mathrm{v})$ heat-inactivated fetal bovine serum (FBS), 1X penicillin/streptomycin and $2 \mathrm{mM}$ L-glutamine (all purchased from EuroClone SpA, Milan, Italy). The cells were maintained at $37^{\circ} \mathrm{C}$ in a humidified atmosphere with $5 \%$ $\mathrm{CO}_{2}$. All the experiments were carried out when the cells were sub-confluent and mycoplasma-negative.

Isolation of EVs from conditioned medium. CABA I cells were starved in serum-free medium for $24 \mathrm{~h}$. The cells were subsequently stimulated with $5 \%$ of $40 \mathrm{~nm}$-filtered FBS HyClone (Thermo Scientific, Rockford, IL, USA) in RPMI-1640 and conditioned medium was collected after the specified time interval for each experiment (30 min and 4, 8 and $18 \mathrm{~h}$ ). For every time-point the same number of cells was seeded at the beginning of experiments.

To isolate EVs, the conditioned medium obtained as above was centrifuged at $600 \mathrm{x}$ g for $15 \mathrm{~min}$ and then at $1,500 \mathrm{x} \mathrm{g}$ for $30 \mathrm{~min}$ to remove cells and large debris, respectively.
Supernatants were centrifuged at $100,000 \mathrm{x}$ for $2 \mathrm{~h}$ at $4^{\circ} \mathrm{C}$ in a Beckmann ultracentrifuge. Isolated vesicles were resuspended in Dulbecco's phosphate-buffered saline (EuroClone, Milan, Italy). Double determination of vesicle quantification was carried out by measuring the vesicle-associated protein levels using the Bradford method (Bio-Rad, Milan, Italy) with bovine serum albumin (Sigma-Aldrich, St. Louis, MO, USA) as the standard.

Electron microscopy. Scanning electron microscopy (SEM) was carried out on subconfluent cells grown on coverslips and fixed with $2 \%$ glutaraldehyde (Electron Microscopy Sciences, Hatfield, PA, USA) in phosphate-buffered saline (PBS) for $30 \mathrm{~min}$. After being critical point-dried, the samples were glued onto stubs, coated with gold in a SCD040 Balzer Sputterer, and detected via a Philips 505 SEM at $20 \mathrm{kV}$.

Transmission electron microscopy (TEM) was performed on isolated vesicles, resuspended in PBS, to analyze their ultrastructural morphology. According to the proper dilutions, the samples were adsorbed onto 300 mesh carbon-coated copper grids (Electron Microscopy Sciences) for $5 \mathrm{~min}$ in a humidified chamber at room temperature. Vesicles on grids were then fixed in 2\% glutaraldehyde (Electron Microscopy Sciences) in PBS for $10 \mathrm{~min}$ and then briefly rinsed in Milli-Q water. Grids with adhered vesicles were examined with a Philips CM 100 transmission electron microscope operating at $80 \mathrm{kV}$, after negative staining with $2 \%$ phosphotungstic acid, brought to $\mathrm{pH} 7.0$ with $\mathrm{NaOH}$. Images were captured with a Kodak digital camera.

NanoSight. The number and dimension of EVs were assessed by the nanoparticle tracking analysis (NTA). Using a NanoSight LM10-HS system (NanoSight Ltd., Amesbury, UK), EVs were visualized by laser light scattering. Briefly, EV-enriched pellets were resuspended in $300 \mu \mathrm{l}$ of $0.1 \mu \mathrm{m}$ triple-filtered sterile PBS and five recordings of $30 \mathrm{sec}$ were performed for each sample. EVs pellets were derived from an equal volume of conditioned medium, that was collected from cells originally seeded in the same number.

Collected data were analyzed with NTA software, which provided high-resolution particle size distribution profiles and concentration measurements of the vesicles in solution.

Western blot analysis. Vesicles ( $8 \mu \mathrm{g}$ for CD63, $40 \mu \mathrm{g}$ for HLA and Ago-2) were resolved by $10 \%$ sodium dodecyl sulfatepolyacrylamide gel electrophoresis (SDS-PAGE) under non-reducing conditions without heating and transferred to nitrocellulose membranes (Whatman-GE Healthcare Life Sciences, UK). Non-specific binding sites were blocked for $1 \mathrm{~h}$ in $10 \%$ non-fat dry milk in TBS containing $0.5 \%$ Tween-20 (TBS-T) at room temperature. The blots were then incubated at $4^{\circ} \mathrm{C}$ overnight with primary antibodies against CD63 (mouse monoclonal, 1:500 dilution; sc-59286), against the W6/32 antigenic determinant of HLA (mouse monoclonal, 1:500 dilution; sc-32235) (both from Santa Cruz Biotechnology, Inc., Santa Cruz, CA, USA) and against Ago-2 (mouse monoclonal, 1:200 dilution; 011-22033, Wako, Japan). Normalization was carried out using the GAPDH antibody (mouse monoclonal, 1:5,000 dilution; MA5-11114, Thermo Scientific, Rockford, IL, USA). 

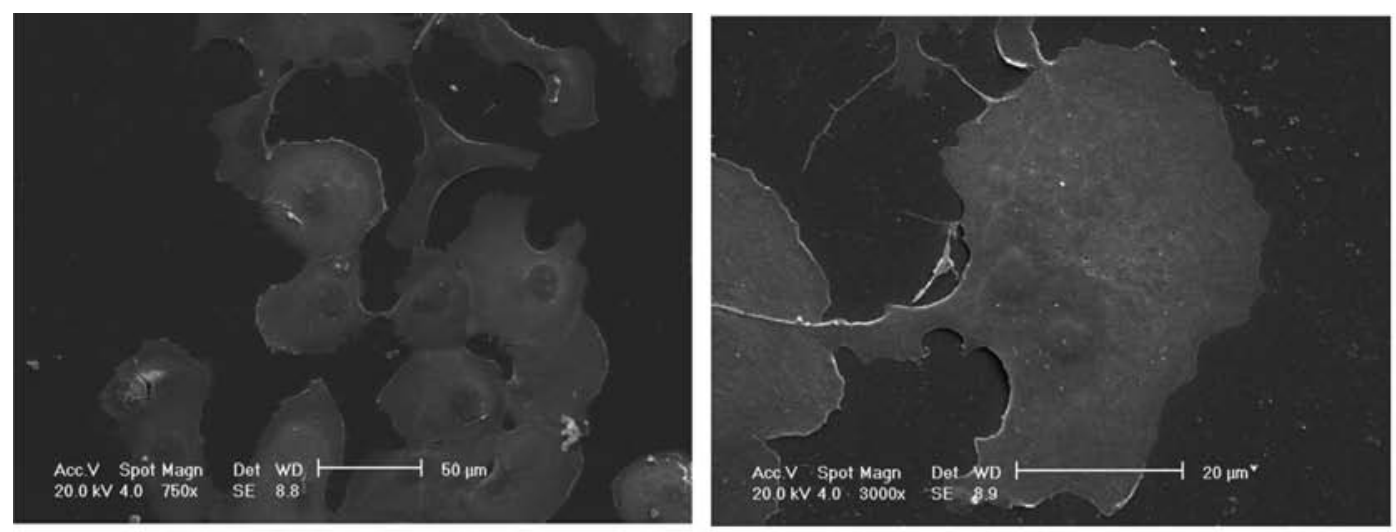

\section{0 min stimulation}
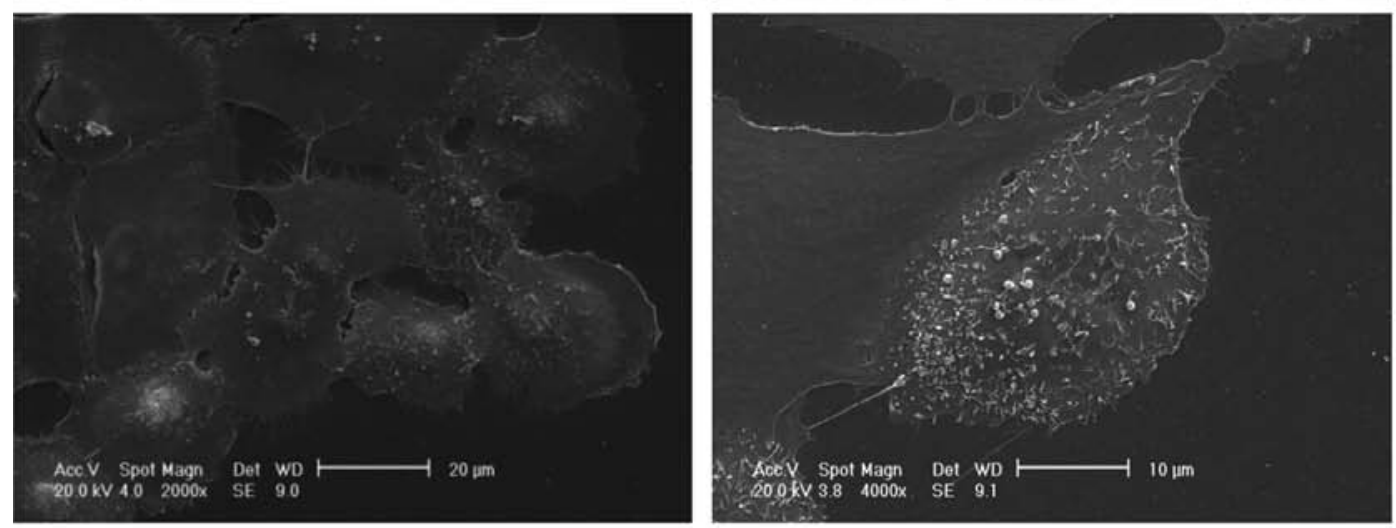

$4 \mathrm{~h}$ stimulation
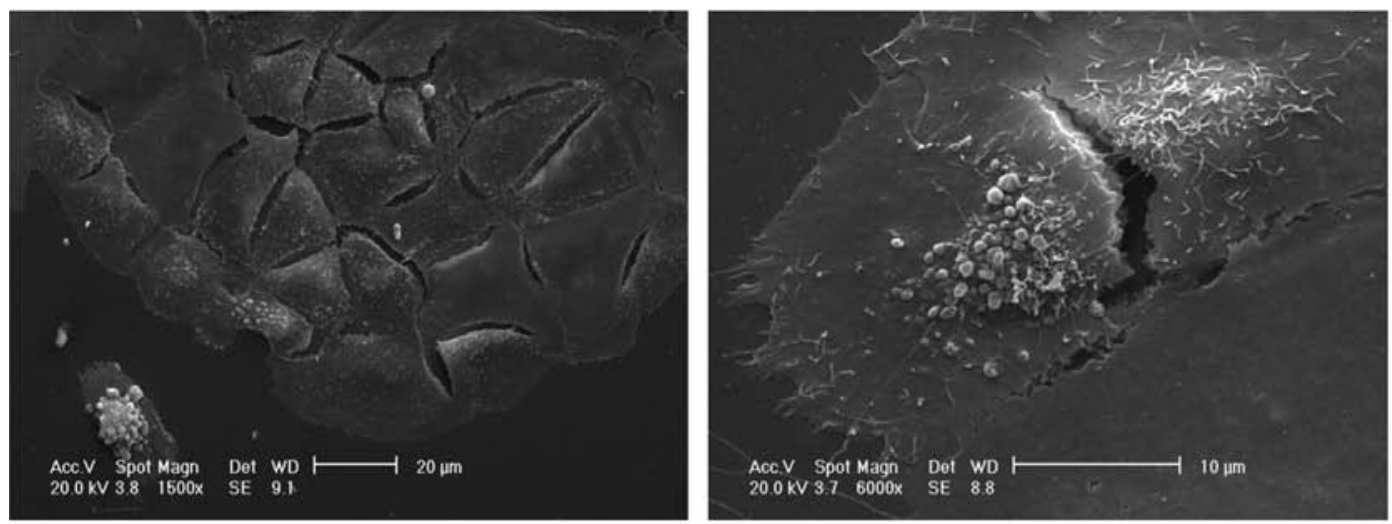

\section{$8 \mathrm{~h}$ stimulation}
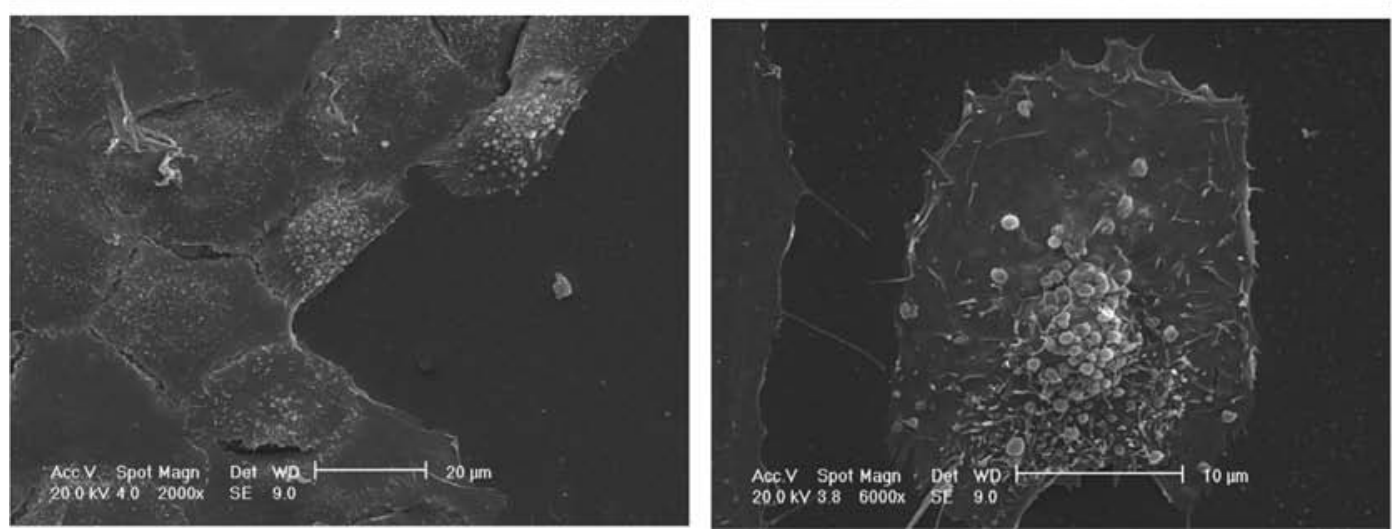

\section{$18 \mathrm{~h}$ stimulation}

Figure 1. SEM analysis. SEM images of CABA I at $30 \mathrm{~min}$ and 4-, 8- and 18-h post-FBS stimulation. SEM, scanning electron microscopy.

After several washes in TBS-T, the membranes were incubated in a peroxidase-conjugated secondary antibody (goat anti-mouse IgG-HRP, 1:10,000 dilution; sc-2005; Santa Cruz Biotechnology, Inc.) for $1 \mathrm{~h}$. All the antibodies were diluted in blocking buffer (TBS-T containing $1 \%$ non-fat dry milk).
After washing in TBS-T, the reactive bands were visualized with a chemiluminescence detection kit (SuperSignal West Pico Chemiluminescent Substrate; Thermo Scientific) and images were recorded with the gel documentation system Alliance LD2 (UVItec, Cambridge, UK). 

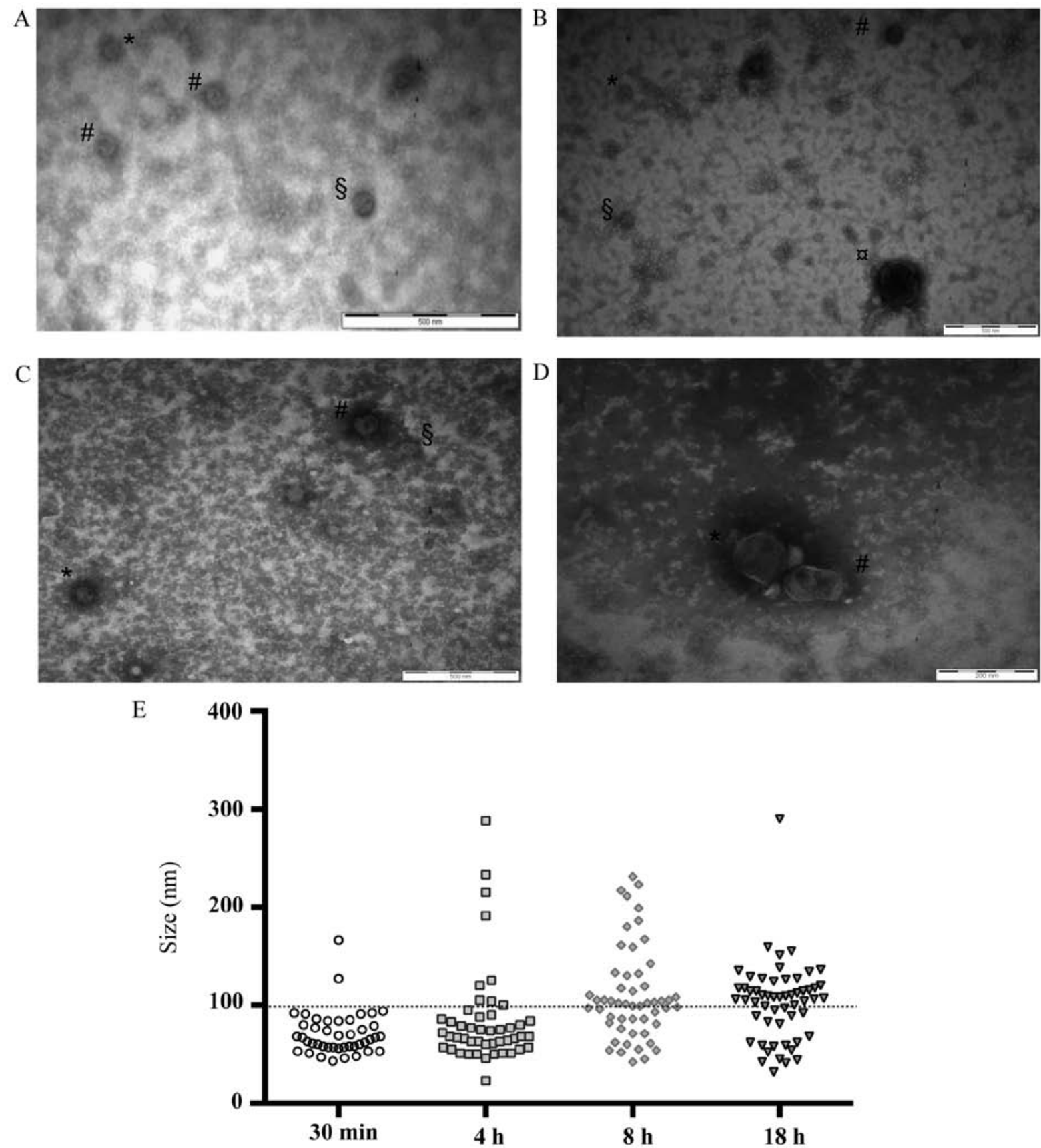

Figure 2. TEM analysis. Transmission electron microscopy images of vesicles isolated from conditioned medium of CABA I cells. (A) Post-FBS stimulation (30 min). Bar, $500 \mathrm{~nm}$ (EVs size: *, $43 \mathrm{~nm} ;{ }^{*}, 51 \mathrm{~nm} ;{ }^{8}, 63 \mathrm{~nm}$ ). (B) Post-FBS stimulation (4 h). Bar, $500 \mathrm{~nm}$ (EVs size: \#, $75 \mathrm{~nm} ;{ }^{*}, 66 \mathrm{~nm} ;{ }^{8}, 51 \mathrm{~nm} ;{ }^{\circ}, 233 \mathrm{~nm}$ ) (C) Post FBS stimulation (8 h). Bar, 500 nm (EVs size: " , 104 nm; ;, 81 nm; $\left.{ }^{8}, 52 \mathrm{~nm}\right)$. (D) Post-FBS stimulation (18 h). Bar, $200 \mathrm{~nm}$ (EVs size: ", $126 \mathrm{~nm}$; *, $114 \mathrm{~nm}$ ). (E) Graph shows the size of EVs analyzed from 19-20-19-34 transmission electron microscopy images (for 30 min, 4,8 and $18 \mathrm{~h}$, respectively): each $\mathrm{EV}$ observed on the grids corresponds to one dot. The horizontal bar is the size limit $(100 \mathrm{~nm})$ that usually distinguishes exosomes and microvesicle subpopulations. TEM, transmission electron microscopy; EVs, extracellular vesicles.

The optical density of the target bands was determined using the Alliance LD2 gel documentation system or the ImageJ public domain software.

Zymography assays. Gelatin zymography was performed using SDS-PAGE (7.5\%) copolymerized with $2 \mathrm{mg} / \mathrm{ml}$ gelatin type B (Sigma-Aldrich). Vesicles $(10 \mu \mathrm{g})$ were diluted in SDS-PAGE sample buffer under non-reducing conditions without heating. After electrophoresis, the gels were washed three times, 15 min each, with $50 \mathrm{mM}$ Tris- $\mathrm{HCl}$ ( $\mathrm{pH}$ 7.4) containing 2.5\% Triton X-100 (Sigma-Aldrich) under agitation at room temperature and then incubated overnight in collagenase buffer [50 mM Tris- $\mathrm{HCl}(\mathrm{pH}$ 7.4) containing $120 \mathrm{mM}$ $\mathrm{NaCl}, 5 \mathrm{mM} \mathrm{CaCl}]_{2}$ at $37^{\circ} \mathrm{C}$. The gels were stained with $0.25 \%$ Coomassie Brilliant Blue R-250 (Bio-Rad) in a mixture of methanol-acetic acid-water (4:1:5) for $30 \mathrm{~min}$ and were destained in the same solution without dye. The gelatinase activities were visualized as distinct white bands on a blue background, indicating proteolysis of the substrate.

Casein-plasminogen zymography was performed on vesicles $(8 \mu \mathrm{g})$ diluted in SDS-PAGE sample buffer under non-reducing conditions without heating, using SDS-PAGE (10\%) gels 

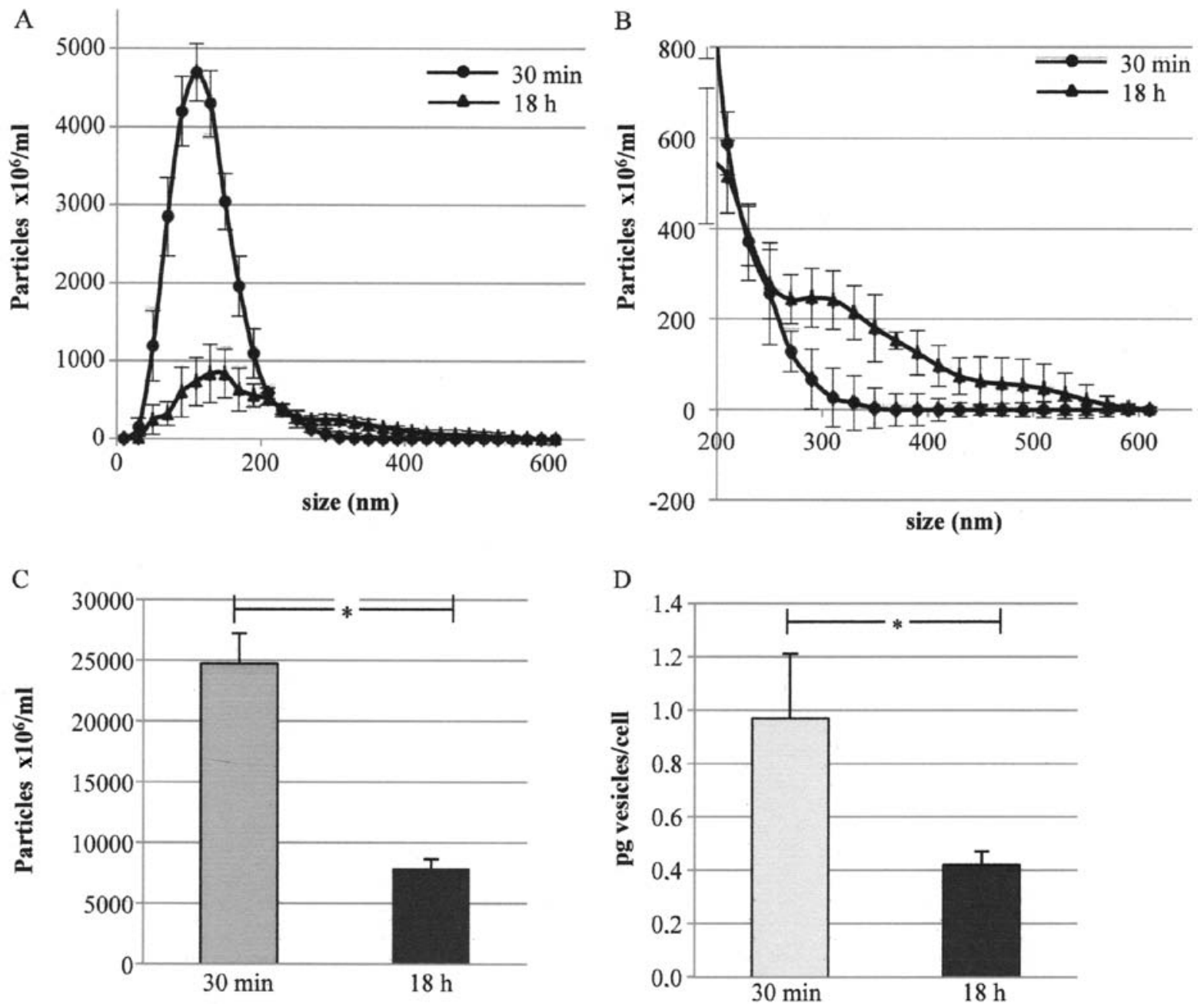

Figure 3. Size measurement and quantification of EVs. (A) Size distribution of CABA I particles (i.e., EVs) assessed by NanoSight assay at $30 \mathrm{~min}$ and $18 \mathrm{~h}$ post-FBS stimulation. (B) The same graph as in (A) reporting a size $>200 \mathrm{~nm}$. (C) Count of total number of particles contained in the two conditioned media as calculated by NanoSight analysis. (D) Quantification of EVs in the conditioned medium. The image shows the amount of EVs released from CABA I cells at $30 \mathrm{~min}$ and $18 \mathrm{~h}$ post-FBS stimulation (expressed as pg released/cell). Data were performed in triplicate $(\mathrm{n}=3)$ and analyzed using the Student's t-test $(\mathrm{P}<0.05)$. Error bars correspond to standard deviation. EVs, extracellular vesicles.

copolymerized with $0.2 \%$ casein plus $10 \mu \mathrm{g} / \mathrm{ml}$ plasminogen (both from Sigma-Aldrich). After electrophoresis, the gels were washed twice for $30 \mathrm{~min}$ in Tris $50 \mathrm{mM} \mathrm{pH} 7.4+$ Triton $\mathrm{X}$ $2.5 \%$ at room temperature and incubated overnight in the same buffer without Triton $\mathrm{X}$ at $37^{\circ} \mathrm{C}$. Gel staining and destaining was performed as for the gelatin zymography.

Statistical analysis. Data shown are from at least three independent experiments and are presented as mean \pm SD. Statistical significance was determined using the Student's t-test. Calculations were performed using GraphPad Prism 4 software (GraphPad, San Diego, CA, USA). Statistical significance was set at $\mathrm{P}<0.05$.

\section{Results}

Time-dependent release of EV subpopulations. In the preliminary experiments the EVs release from starved cells after FBS stimulation was evaluated by means of SEM and TEM.

The SEM assay revealed a time-dependent release of EVs from the cell surface (Fig. 1). After $30 \mathrm{~min}$, the cell surface completely lacked any membrane movement and vesicle budding, whereas after $4 \mathrm{~h}$ membrane movements were evident and vesicle release commenced. The latter phenomenon was particularly evident after $8 \mathrm{~h}$, and even more, after $18 \mathrm{~h}$. The excretion of EVs involved mainly the central portion of the cell body.

TEM analysis of EVs isolated at different time intervals after cell stimulation confirmed their release in the conditioned medium (Fig. 2A-D) and showed that after $30 \mathrm{~min}$ mainly small EVs were released (mean size, $<100 \mathrm{~nm}$ ), while the release of larger EVs (mean size, $>100 \mathrm{~nm}$ ) occurred later (Fig. 2E). Moreover, the amount of smaller EVs decreased as the stimulation time increased, whereas the opposite occurred for the larger EVs, whose presence was recorded after $4 \mathrm{~h}$ and whose amount increased proportionally to the stimulation time (Fig. 2E).

In the subsequent experiments only the shorter and longer stimulation time were taken into account. EVs released in medium after $30 \mathrm{~min}$ and $18 \mathrm{~h}$ from starved cells after FBS stimulation were isolated and their number and size distribution were analyzed by means of NanoSight. Fig. 3A shows the number of particles in the conditioned media after deduction of the contribution of particles from non-conditioned ones. Fig. 3B 

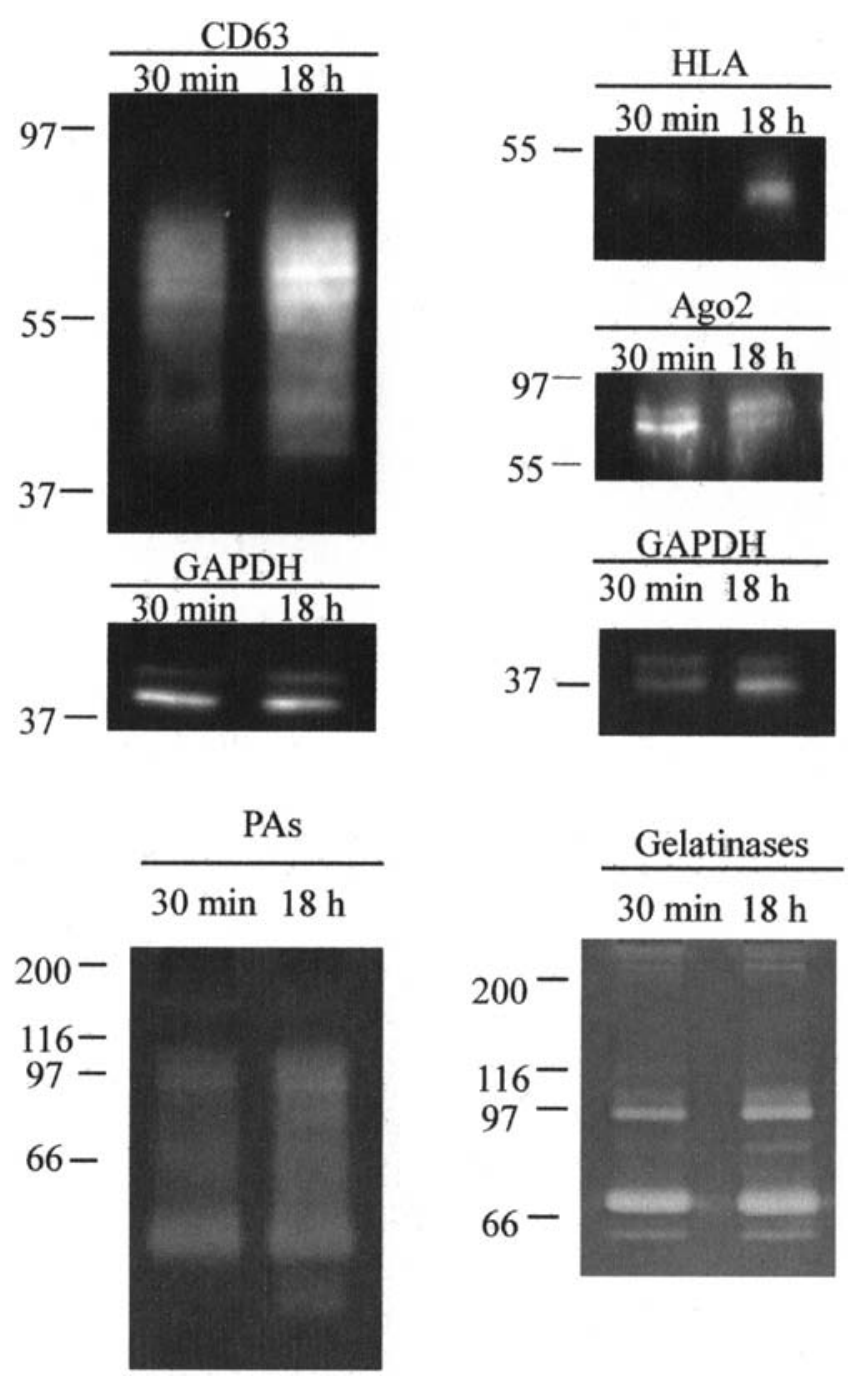

Figure 4. Molecular characterization. At $30 \mathrm{~min}$ and $18 \mathrm{~h} \mathrm{EV} \mathrm{subpopulations}$ CD63, HLA and Ago-2 (upper panels) were analyzed by western blotting whereas gelatinases and plasminogen activators (PAs) (lower panels) were analyzed by means of gelatin and casein-plasminogen zymography, respectively. Molecular weights are exhibited on the left of each image. For western blotting the total GAPDH signal for the two samples (30 min and $18 \mathrm{~h}$ ) measured by means of densitometric assay was equivalent. EVs, extracellular vesicles.

also shows the same graph reporting only a size of $>200 \mathrm{~nm}$. After 30 min the cells released mainly particles whose mean size was $\sim 100 \mathrm{~nm}$ (peak mean in five replicates at 100-127 nm) whereas after $18 \mathrm{~h}$ an additional peak at higher dimensions was evident (lower and higher peak mean in five replicates at 126-135 and 371-456 nm, respectively) (Fig. 3A and B).

The NanoSight assay also showed that the number of particles (i.e., of EVs) was higher in the medium conditioned for $30 \mathrm{~min}$ than that in the medium conditioned for $18 \mathrm{~h}\left(24,712 \times 10^{6} / \mathrm{ml}\right.$ and $7,857 \times 10^{6} / \mathrm{ml}$ particles, respectively) (Fig. 3C). A further quantification performed by the analysis of EVs protein content confirmed the previous data: the amount of EVs was higher in the medium conditioned for $30 \mathrm{~min}$ than that in the medium conditioned for $18 \mathrm{~h}(0.97$ and $0.42 \mathrm{pg} / \mathrm{cell}$, respectively) (Fig. 3D).

Molecular characterization. CD63, HLA and Ago-2 expression was assessed using western blotting. The presence of gelatinases and plasminogen activators was also assessed by zymography techniques (Fig. 4).

The anti-CD63 antibody revealed a protein with a size ranging 39-77 $\mathrm{kDa}$, suggesting that the CD63 contained in vesicles was in a glycosylated form. In EVs released after $18 \mathrm{~h}$ stimulation the amount of protein was $\sim 2$-fold higher $(1.92 \pm 0.12)$ than that in EVs released after $30 \mathrm{~min}$.

The anti-HLA antibody revealed a protein with a size of $50 \mathrm{kDa}$. In EVs released after $18 \mathrm{~h}$ stimulation the amount of protein was $\sim 3$-fold higher $(2.84 \pm 0.28)$ than that in EVs released after $30 \mathrm{~min}$.

Anti-Ago2 revealed a protein with a size of $78-93 \mathrm{kDa}$ that was slightly more expressed in EVs released after $30 \mathrm{~min}$ (expression $\sim 1.2$ higher in $30 \mathrm{~min}, 1.25 \pm 0.13$ ).

Gelatin zymography allowed for the identification of the gelatinases MMP-2 and - 9 both in the pro-enzyme and active form in the two EVs samples (calculated molecular weights: proMMP-9, 97 kDa; MMP-9, 84 kDa; proMMP-2, $72 \mathrm{kDa}$; and MMP-2, $64 \mathrm{kDa}$ ). MMPs complexes and/or dimers were also present at a high molecular weight. The amount of enzymes was substantially equal in the two samples, with the exception of a slight increase of proMMP-9 and MMP-9 in the EVs released after $18 \mathrm{~h}$ stimulation.

Casein-plasminogen zymography showed the presence of plasminogen activators in EVs. The assay emphasized that PA-PAI complexes (84-102 kDa), tissue type-PA (67 kDa) and urokinase type-PA (both uPA precursor, $55 \mathrm{kDa}$; and active uPA, 33 kDa-forms) were associated with EVs. Additionally, the amount of enzymes was substantially equal in the two samples, with the exception of the LMW-uPA, which was only present in EVs released after $18 \mathrm{~h}$ stimulation.

\section{Discussion}

At present, an ongoing challenge in the study of EVs is to identify and standardize a method that enables a careful separation of EVs subpopulations released by cells in conditioned medium (in vitro) and in biological fluids (in vivo) (1). In fact, even if EXOs and MVs clearly differ in their size ranges and cell origin, their specific features as well as specific functions are not completely clear since the available isolation techniques are not able to separate the subpopulations in a precise manner (1). This is probably due to the size overlap of subpopulations and the lack of specific markers. Two main strategies are used for the separation of EVs subpopulations, based on their physical properties or biochemical features that, however, seem insufficient for a clear separation (1).

In the former approach, size and density are used as reference parameters and this strategy involves the use of serial centrifugations and flotation in sucrose gradients (4).

These methods, being based on physical characteristics, have the advantage of not requiring previous knowledge of chemical signatures of EVs and the disadvantage of not allowing the complete discrimination between EXOs and MVs (since the size distribution of MVs overlaps with that of EXOs at its lower limit, thus it is possible that these conditions pellet mixed vesicle populations). For this reason, it may be useful to combine differential centrifugation with sucrose gradient ultracentrifugation to isolate EXOs subpopulation or to proceed by immunoisolation which requires, however, a 
preliminary knowledge of the EVs marker profiles (1). In the latter approach, magnetophoretic sorting or immunoaffinity chromatography are used (4). However, no 'optimal' method has been identified thus far (30).

With regard to EVs quantification cytometry is the most widely used method as it uses size and affinity measurements through conjugation with specific fluorescent antibodies. However, that vesicles $<200 \mathrm{~nm}$ cannot be distinguished from instrumental noise must be taken into account. Thus, EXOs and smaller MVs cannot be detected using this technique $(8,31)$. However, a fluorescence-based, high-resolution flow cytometric method has been previously developed for quantitative and qualitative analysis of nanosized membrane vesicles (32). A novel strategy based on the differential light scattering of different size particles solved in a fluid medium (NanoSight) has also been previously employed to count and evaluate the size distribution of nanoparticles (4).

A clear separation of the two subpopulations may allow us to understand specific features of each subgroup. In order to obtain this aim, a deeper knowledge of mechanisms involved in EVs release may be useful to understand their biology, an absolute requisite to decode their specific role in both physiological and pathological conditions. Additionally, standardization of procedures is required to allow a proper comparison of data obtained from different laboratories, as previously established (30).

Thus we examined whether different EV subpopulations were released in a time-dependent manner using CABA I cells that were first starved in order to synchronize cells and to block EVs release. FBS deprivation is known to inhibit MVs shedding (33) and we confirmed that it also inhibits EXOs release, as identified by the fact that no EVs were recovered from the conditioned medium of starved CABA I (data not shown).

After starvation, the cells were stimulated with FBS and conditioned medium was collected after different time intervals. The shorter one was set at 30 min since it is known that $90 \%$ of EXOs are externalized within a short time period from stimulation (34). The subsequent intervals were set at 4 , 8 and $18 \mathrm{~h}$. The result from the SEM assay suggested that EVs release is a gradual process that increases over time as shown by the fact that no vesicles or membrane movement were visible after $30 \mathrm{~min}$ although their presence increased over time and reached the maximum after $18 \mathrm{~h}$. The TEM analysis of isolated vesicles revealed that in $30 \mathrm{~min}$ the population of EVs was represented almost exclusively by EVs $<100 \mathrm{~nm}$ (most likely EXOs) while the presence of larger EVs (most likely MVs) was very sporadic. The number of MVs increased over time, with a low number of MVs after 4 and $8 \mathrm{~h}$ and a larger quantity after $18 \mathrm{~h}$. At the latter time, however, EXOs were evident although more infrequently when compared to $30 \mathrm{~min}$. Thus, when the cells were stimulated EXOs were rapidly externalized, as they were already stored inside the cell in multivesicular bodies, while the cell membrane movements leading to MVs release required a longer time period.

Thus, we focused on EVs released after $30 \mathrm{~min}$ and $18 \mathrm{~h}$ after stimulation, corresponding to the population with the higher amount of EXOs and the population with a higher amount of MVs combined with the lower EXOs contribution, respectively.
This finding was further evidenced with the NanoSight assay, suggesting the presence of mainly EXOs in the medium conditioned for $30 \mathrm{~min}$ and the subpopulations of EXOs and MVs in the medium conditioned for $18 \mathrm{~h}$, although in the latter the amount of smaller EVs was lower (as underlined by the minor peak height). It is known that NanoSight is unable to distinguish EVs from non-membranous particles of similar size (30). Thus, non-conditioned media were treated simultaneously with and in exactly the same manner as conditioned media and were used as a blank. Moreover, EVs were resuspended in $0.1 \mu \mathrm{m}$ triple-filtered PBS to remove any possible interfering elements, PBS was used as the blank.

NanoSight and the quantification performed by analysis of EVs protein content suggested that the amount of total EVs was higher after $30 \mathrm{~min}$ than after $18 \mathrm{~h}$. We cannot explain the reason for the amount of EVs decreasing over time, but we hypothesize that some of them break or fuse with target cells.

We assessed the presence of several proteins in our preparations to determine whether any molecules were differentially expressed. For a generic characterization of EVs, the International Society for Extracellular Vesicles (ISEV) suggests the investigation of several proteins from various categories including extracellular, intracellular, transmembrane and cytosolic (30). We therefore analyzed two transmembranes (CD63 and HLA), one intracellular (Ago-2) and two extracellular (gelatinases and plasminogen activators) proteins. CD63, particularly, was long considered a specific EXOs marker, but not more nowadays (35-37). CD63 and HLA expression is more evident in EVs obtained after $18 \mathrm{~h}$ of stimulation as compared to Ago-2, which seems to be slightly more expressed in EVs obtained after $30 \mathrm{~min}$. By contrast, gelatinases and plasminogen activators activity of EVs was similar in the two samples, even if after $18 \mathrm{~h}$ the active form of MMP-9 and uPA was markedly expressed. It should be noted, moreover, that CD63 is contained in vesicles in a glycosylated form. It is known that glycosylation levels of CD63 strongly influence its cell surface expression and it has been shown that the glycosylation-mediated localization of CD63 is associated with several features of cancer progression, such as drug resistance and invasiveness (38).

Results of the present study suggest that different times lead to release of different subpopulations of EVs, in terms of size, amount and molecular composition. Additionally, the stimulation time should be considered an important factor when searching among parameters that are useful in the standardization of procedures for EVs isolation and analysis. Thus, the time between the stimulation of cells and collection of conditioned media in vitro, and to some extent in vivo, affects the heterogeneity and variability of the results. It is therefore imperative to consider and select the appropriate time point to collect samples following any treatment in clinical studies.

\section{References}

1. Raposo G and Stoorvogel W: Extracellular vesicles: exosomes, microvesicles, and friends. J Cell Biol 200: 373-383, 2013.

2. Ohno S, Ishikawa A and Kuroda M: Roles of exosomes and microvesicles in disease pathogenesis. Adv Drug Deliv Rev 65: 398-401, 2013. 
3. Turturici G, Tinnirello R, Sconzo G and Geraci F: Extracellular membrane vesicles as a mechanism of cell-to-cell communication: advantages and disadvantages. Am J Physiol Cell Physiol 306: C621-C633, 2014.

4. D'Souza-Schorey Cand Clancy JW: Tumor-derived microvesicles: shedding light on novel microenvironment modulators and prospective cancer biomarkers. Genes Dev 26: 1287-1299, 2012.

5. Enjeti AK, Lincz LF and Seldon M: Microparticles in health and disease. Semin Thromb Hemost 34: 683-691, 2008.

6. Rak J: Microparticles in cancer. Semin Thromb Hemost 36: 888-906, 2010.

7. Mathivanan S, Ji H and Simpson RJ: Exosomes: extracellular organelles important in intercellular communication. J Proteomics 73: 1907-1920, 2010.

8. György B, Módos K, Pállinger E, Pálóczi K, Pásztói M, Misják P, Deli MA, Sipos A, Szalai A, Voszka I, et al: Detection and isolation of cell-derived microparticles are compromised by protein complexes due to shared biophysical parameters. Blood 117: e39-48, 2011

9. Bobrie A, Colombo M, Raposo G and Thery C: Exosome secretion: molecular mechanisms and roles in immune responses. Traffic 12: 1659-1668, 2009.

10. Taraboletti G, D’Ascenzo S, Borsotti P, Giavazzi R, Pavan A and Dolo V: Shedding of the matrix metalloproteinases MMP-2, MMP-9, and MT1-MMP as membrane vesicle-associated components by endothelial cells. Am J Pathol 160: 673-680, 2002.

11. Van Niel G, Raposo G, Candalh C, Boussac M, Hershberg R, Cerf-Bensussan $\mathrm{N}$ and Heyman M: Intestinal epithelial cells secrete exosome-like vesicles. Gastroenterology 121: 337-349, 2001.

12. Krämer-Albers EM, Bretz N, Tenzer S, Winterstein C, Möbius W, Berger H, Nave KA, Schild H and Trotter J: Oligodendrocytes secrete exosomes containing major myelin and stress-protective proteins: trophic support for axons? Proteomics Clin Appl 1: 1446-1461, 2007.

13. Fauré J, Lachenal G, Court M, Hirrlinger J, Chatellard-Causse C, Blot B, Grange J, Schoehn G, Goldberg Y, Boyer V, et al: Exosomes are released by cultured cortical neurones. Mol Cell Neurosci 31: 642-648, 2006.

14. Dolo V, D'Ascenzo S, Giusti I, Millimaggi D, Taraboletti G and Pavan A. Shedding of membrane vesicles by tumor and endothelial cells. Ital J Anat Embryol 110: 127-133, 2005.

15. Hood JL, San RS and Wickline SA: Exosomes released by melanoma cells prepare sentinel lymph nodes for tumo metastasis. Cancer Res 71: 3792-3801, 2011.

16. Al-Nedawi K, Meehan B and Rak J: Microvesicles: Messengers and mediators of tumor progression. Cell Cycle 8: 2014-2018, 2009.

17. Muralidharan-Chari V, Clancy JW, Sedgwick A and D'Souza-Schorey C: Microvesicles: mediators of extracellular communication during cancer progression. J Cell Sci 123: $1603-1611,2010$

18. Lee TH, D'Asti E, Magnus N, Al-Nedawi K, Meehan B and Rak J: Microvesicles as mediators of intercellular communication in cancer - the emerging science of cellular 'debris.' Semin Immunopathol 33: 455-467, 2011.

19. Giusti I, D'Ascenzo S and Dolo V: Microvesicles as potential ovarian cancer biomarkers. Biomed Res Int 2013: 703048, 2013.

20. Mause SF and Weber C: Microparticles: protagonists of a novel communication network for intercellular information exchange. Circ Res 107: 1047-1057, 2010.

21. van der Meel R, Krawczyk-Durka M, van Solinge WW and Schiffelers RM: Toward routine detection of extracellular vesicles in clinical samples. Int J Lab Hematol 36: 244-253, 2014

22. Katsuda T, Kosaka N and Ochiya T: The roles of extracellular vesicles in cancer biology: toward the development of novel cancer biomarkers. Proteomics 14: 412-425, 2014.
23. Redzic JS, Ung TH and Graner MW: Glioblastoma extracellular vesicles: reservoirs of potential biomarkers. Pharmgenomics Pers Med 7: 65-77, 2014.

24. Perez A, Loizaga A, Arceo R, Lacasa I, Rabade A, Zorroza K, Mosen-Ansorena D, Gonzalez E, Aransay AM, Falcon-Perez JM, et al: A pilot study on the potential of RNA-associated to urinary vesicles as a suitable non-invasive source for diagnostic purposes in bladder cancer. Cancers (Basel) 6: 179-192, 2014.

25. Hessvik NP, Sandvig K and Llorente A: Exosomal miRNAs as biomarkers for prostate cancer. Front Genet 4: 36, 2013.

26. Duijvesz D, Luider T, Bangma CH and Jenster G: Exosomes as biomarker treasure chests for prostate cancer. Eur Urol 59: 823-831, 2011.

27. Rabinowits G, Gerçel-Taylor C, Day JM, Taylor DD and Kloecker GH: Exosomal microRNA: a diagnostic marker for lung cancer. Clin Lung Cancer 10: 42-46, 2009.

28. Tavoosidana G, Ronquist G, Darmanis S, Yan J, Carlsson L, Wu D, Conze T, Ek P, Semjonow A, Eltze E, et al: Multiple recognition assay reveals prostasomes as promising plasma biomarkers for prostate cancer. Proc Natl Acad Sci USA 108 8809-8814, 2011.

29. Dolo V, Ginestra A, Violini S, Miotti S, Festuccia C, Miceli D, Migliavacca M, Rinaudo C, Romano FM, Brisdelli F, et al: Ultrastructural and phenotypic characterization of CABA I, a new human ovarian cancer cell line. Oncol Res 9: 129-138, 1997.

30. Lötvall J, Hill AF, Hochberg F, Buzás EI, Di Vizio D, Gardiner C, Gho YS, Kurochkin IV, Mathivanan S, Quesenberry P, et al: Minimal experimental requirements for definition of extracellular vesicles and their functions: a position statement from the International Society for Extracellular Vesicles. J Extracell Vesicles 3: 26913, 2014.

31. Lacroix R, Robert S, Poncelet P, Kasthuri RS, Key NS and Dignat-George F: Standardization of platelet-derived microparticle enumeration by flow cytometry using calibrated beads: results of ISTH SSC collaborative workshop. J Thromb Haemost 8: 2571-2574, 2010.

32. Nolte-'t Hoen EN, van der Vlist EJ, Aalberts M, Mertens HC, Bosch BJ, Bartelink W, Mastrobattista E, van Gaal EV, Stoorvogel W, Arkesteijn GJ, et al: Quantitative and qualitative flow cytometric analysis of nanosized cell-derived membrane vesicles. Nanomedicine 8: 712-720, 2012.

33. Dolo V, Ginestra A, Cassarà D, Violini S, Lucania G, Torrisi MR, Nagase H, Canevari S, Pavan A and Vittorelli ML: Selective localization of matrix metalloproteinase 9, betal integrins, and human lymphocyte antigen class I molecules on membrane vesicles shed by $8701-\mathrm{BC}$ breast carcinoma cells. Cancer Res 58: 4468-4474, 1998.

34. Koumangoye RB, Sakwe AM, Goodwin JS, Patel T and Ochieng J: Detachment of breast tumor cells induces rapid secretion of exosomes which subsequently mediate cellular adhesion and spreading. PLoS One 6: e24234, 2011.

35. Grant R, Ansa-Addo E, Stratton D, Antwi-Baffour S, Jorfi S, Kholia S, Krige L, Lange S and Inal J: A filtration-based protocol to isolate human plasma membrane-derived vesicles and exosomes from from blood plasma. J Immunol Methods 371: 143-151, 2011.

36. Witwer KW, Buzás EI, Bemis LT, Bora A, Lässer C, Lötvall J, Nolte-'t Hoen EN, Piper MG, Sivaraman S, Skog J, et al: Standardization of sample collection, isolation and analysis methods in extracellular vesicles research. J Extracell Vesicles 2: 20360, 2013

37. Andreu Z, Yáñez-Mó M: Tetraspanins in extracellular vesicles formation and function. Front Immunol 5: 442, 2014.

38. Tominaga N, Hagiwara K, Kosaka N, Honma K, Nakagama H, Ochiya T: RPN2-mediated glycosylation of tetraspanin CD63 regulates breast cancer cell malignancy. Mol Cancer 13: 134, 2014. 